\title{
Current Conduction in Dual Channel Black Phosphorene Nanoribbon Transistor
}

\author{
Zuriana Auzar, Zaharah Johari* and Nurul Ezaila Alias \\ School of Electrical Engineering, Faculty of Engineering, Universiti Teknologi Malaysia, 81310 UTM Johor Bahru \\ Johor, Malaysia
}

*Corresponding author: zaharahj@utm.my

\begin{abstract}
Phosphorene continues to fascinate research community due to its excellent physical and electrical properties. In this paper, the feasibility study of using dual conducting channel in black phosphorene nanoribbon transistor is investigated using Atomistic simulation tool. Both electronic and transport properties are evaluated. Through simulation, it is demonstrated that the conduction behavior behave differently where the current exhibit a great deal of increment when using dual-channel. The performance was superior compared to single-channel and suggests that the number of conducting channel is a significant factor in improving device behavior.
\end{abstract}

Keywords: black phosphorene, electronic properties, dual-channel phosphorene

(C) 2018 Penerbit UTM Press. All rights reserved

Article History: received 2 August 2018; accepted 22 August 2018; published 29 August 2018.

\section{INTRODUCTION}

Throughout the years, researchers explored many alternative materials to sustain the device performance especially when the device dimension has been shrunk to nanoscale. One of the materials is phosphorene. Researchers study on phosphorene since it was isolated in 2014 [1] recognizing its potential uses in the field of electronic applications, such as field-effect transistors [2$5]$ and optoelectronics devices [1, 6, 7]. The high mobility of phosphorene up to $1000 \mathrm{~cm}^{2} / \mathrm{V}$ and the presence of energy bandgap obviate the need to modify its structural morphology [6]. This extraordinary properties of phosphorene held the promise of electronic devices with improved performance while scaling down its dimension. Similar to graphene, monolayer phosphorene structure can in a form of nanoribbon with armchair or zigzag direction on its width. Both structures is referred to armchair phosphorene nanoribbon (APNRs) and zigzag phosphorene nanoribbon (ZPNR). Both structures can be defined by the number of dimer lines for the armchair or the zigzag chains across the ribbon width [8]. Phosphorene nanoribbon reveals that have positive and much smaller formation energies compared to GNR [9].

A number of studies have been undertaken to investigate various phosphorene based field effect transistor (FET). Multiple conducting channel of phosphorene is among the great potential for electronics device. Numerous experiment have established on multiple conducting channel using carbon nanotube (CNT) in FET and graphene nanoribbon (GNR). For example, a local backgated aligned CNT array transistor has been proven to exhibit current density of more than $40 \mathrm{~A} / \mathrm{m}$ [10]. In addition, Franklin et al. [10] reported that the ON current of the array CNT FET scales linearly with number of conducting channel. The practicality of implementing array channel in FET is also evident in array vertical nanowire transistor with gated surround structure where it exhibits improvement in current flowing [11]. The enhancement in FET performance using multiple conducting channel is promising and yet to be demonstrated using APNR. Therefore, in this paper, the feasibility of using multiple conducting channel of APNR in FET is investigated through simulation using the Atomistik Tool Kit from Quantum Wise version 14.2. In the next section, the computational details of the simulation is explained.

\section{METHODOLOGY}

\subsection{Geometric Structure}

The simulation is carried out using VNL-ATK software version 14.2 from Quantum Wise [12]. Crystal builder tool is used in order to build the structure of monolayer black phosphorene. Phosphorous atoms in the bulk form are connected via Van der Waals interaction. Each layer of the phosphorene is comprised of phosphorous atoms with covalent bonding to its three adjacent phosphorous (P) atoms forming puckered honeycomb lattice structure with $\mathrm{sp}^{3}$ hybridization. In the crystal builder plugin, the space group of black phosphorus belongs to orthorhombic with $c$ $m$ c $a$ group. The Bravais lattice of $a, b$ and $c$ was set to $3.3136 \AA, 10.478 \AA$ and $4.3763 \AA$ respectively [13]. For the Wyckoff positions for the $x, y$ and $z$ plane was set to $(0$, 0.10168 , and 0.08056 ) for allowed the atoms to occupy in a lattice belonging to a particular space group [13]. Figure 1(a) shows the black phosphorus crystal obtained from the parameter selected. Next, the manipulation of black phosphorous crystal is done by repeating the number of phosphorous atoms in the $a(x)$ and $b(y)$ directions and namely " $a 4 b 5$ " since the $a$ direction repeated 5 times while 
$b$ direction in 4 times. The illustration of the $a 4 b 5$ structure is shown in Figure 1(b) and armchair phosphorene nanoribbon (APNR) structure is shown in Figure 1(c). After the relaxation process, the bond length of $\mathrm{P}-\mathrm{P}$ atoms increased from $2.22 \AA$ to $2.24 \AA$.

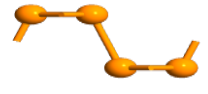

(a)

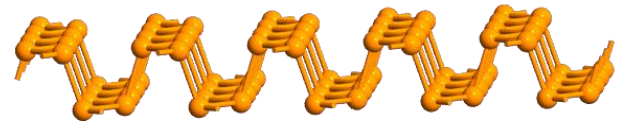

(b)

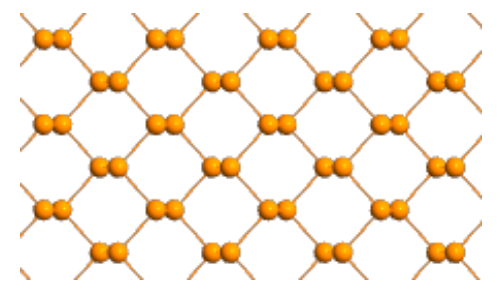

(c)
Figure 1. (a) Black phosphorous crystal and (b) repetition of $a 4 b 5$ structure and (c) armchair phosphorene nanoribbon (APNR).

\subsection{Computational Details}

All the DFT calculations were performed by using the VNL-ATK software version 14.2 from Quantum Wise to study the electronic and transport properties of APNR. The generalized gradient approximation (GGA) with PerdewBurke-Ernzerhof (PBE) functional is utilized. For the electronic calculation, the mesh cut off was 75 Hatree and the $k$-point grid of $1 \times 1 \times 100$ is used. In this software, the $k$-point number at $z$-direction must be restricted to be either equal to be default value, which is 100 or higher for better accuracy. GGA and PBE methods are used in the calculations for the electron exchange correlation. FritzHaber-Institute (FHI) pseudopotential is used with unpolarized spin in the valence electron calculation. Fast Fourier Transform (FFT) and multigrid technique [14] are also used in the electron calculations. Neumann boundary is used in $x$ direction, while periodic boundary is used in both $y$ and $z$ directions.

\section{RESULT AND DISCUSSION}

There are two types of APNRs structure were investigated in this study which are single-channel and dual-channel APNRs. The illustration of the both structures are shown in Figure 2. For the dual-channel, distance between for both channels is $3.7 \AA$. The band structure for black phosphorene is plotted in Figure 3. The separation between the conduction and the valence band of $a 4 b 5$ black phosphorene is $0.81 \mathrm{eV}$ which is close to a study by Carmel et al. [15]. When the layer is made dual, the bandgap reduce to $0.11 \mathrm{eV}$ indicating that the value of bandgap decrease with the increment of number of channel. This is evident from Figure 3(a)-(b), there is a large gap appeared between upper valence band (VB) and bottom conduction band $(\mathrm{CB})$. In contrast to the dual-channel APNR, the gap between the bottom $\mathrm{CB}$ and upper VB is smaller. The same trend of observation was found when increasing the black phosphorene width as shown by Wu et al. [16].

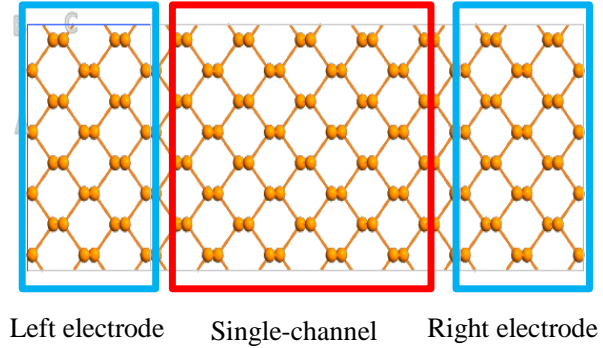

(a)

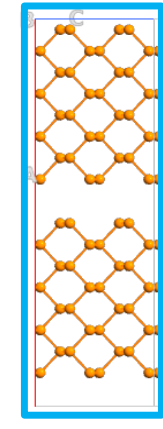

Left electrode

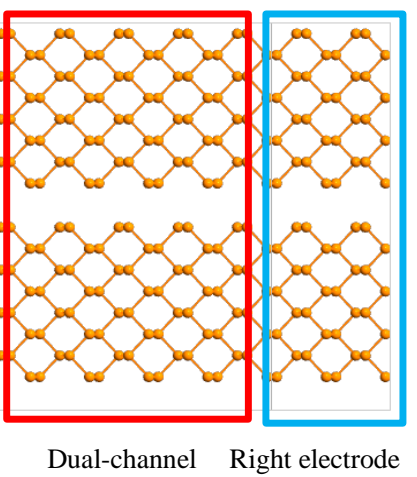

(b)
Figure 2. Front views of APNRs structure; (a) singlechannel and (b) dual-channel.

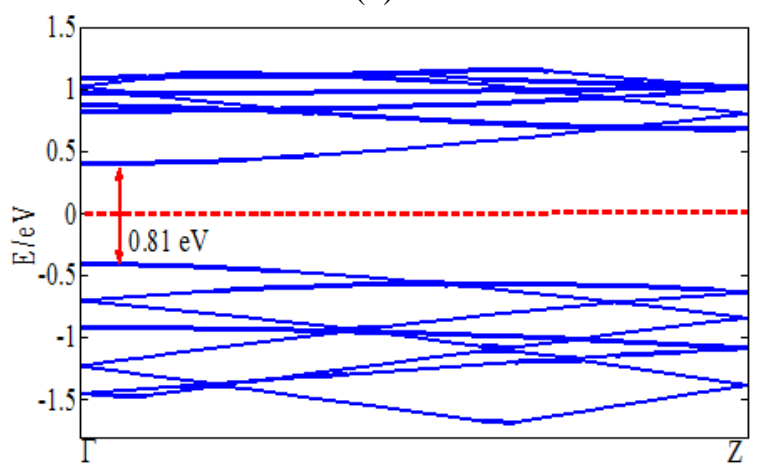

(a)

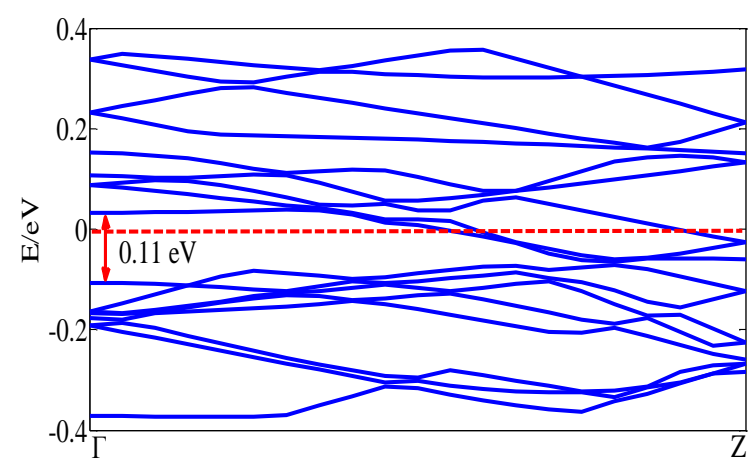

(b)

Figure 3. Bandstructure of APNRs structure for (a) single-channel and (b) dual-channel.

Figure 4 shows the density of state (DOS) for singlechannel and dual-channel APNRs. Referring to Figure 4(a), it clearly shown that, the DOS is zero at the Fermi level. This shows that there is no electron can appear in the forbidden gap. The width of the zero DOS is also approximately the same to the amount value of bandgap. 
In contrast, as shown in Figure 4(b), it can be seen that the width of the zero DOS of dual-channel APNRs is not symmetrical at the Fermi level. This reveals that electron appeared at the Fermi level and localized at the CB and symmetrical at the Fermi level.

This suggest that electron appeared at the Fermi level and localized at the $\mathrm{CB}$ and VB states. This effect can also be viewed from the bandstructure in Figure 3(b) where there are more bands are localized at the valence state compare to conduction state. The DOS for the dualchannel clearly shows how electric field can shift the energy levels to the higher energy and confirms that the field effect transistor-like behavior of PNRs.

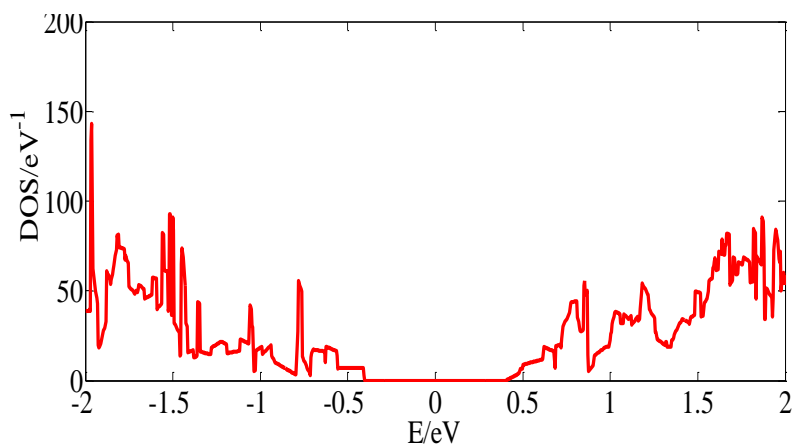

(a)

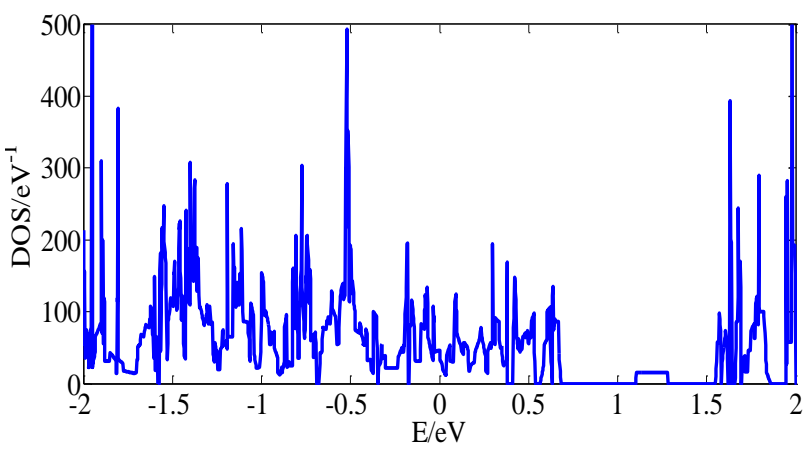

(b)

Figure 4. Density of state of APNRs structure for (a) single-channel and (b) dual-channel.

For the transmission spectrum, $T(E)$ it calculate by using the formula [15],

$$
T(E)=\operatorname{Trace}\left[\Gamma_{L}(E) G^{R}(E) \Gamma_{R}(E) G^{A}(E)\right]
$$

where $\Gamma_{L}(E) \Gamma_{R}(E)$ are the coupling matrix for the left and right electrodes. While $\mathrm{G}^{R}(E)$ is called the retarded and $\mathrm{G}^{A}(E)$ is the advanced Green's function matrix which is given by

$$
G^{R}(E)=[E I-H-\Sigma]^{-1}
$$

where $\sum$ is a non Hermitian matrix which denotes the strength of the source. The system Hamiltonian is represented by $H$. The total current can be calculated from energy dependent transmission function

$$
I=\frac{e}{h} \int T(E)\left(f_{1}-f_{2}\right) d E
$$

where $f_{1}$ and $f_{2}$ are the contact fermi levels. It also similar to the transmission spectrum where dual-channel dominates since transmission coefficient increase at the Fermi level point.

As displayed in Figure 5, the quantized transmission plateaus for the single-channel are suppressed compared to the dual-channel APNR. The suppressed transmission areas for the single-channel APNR at energies -1.88 to $1.52 \mathrm{eV}$ and \pm 0.6 to $\pm 1.4 \mathrm{eV}$ rising sharply when introduced dual-channel APNR. Surprisingly, the energetically transmission plateau appeared is highest with coefficient of 4 at the energy between -1.5 to $-1 \mathrm{eV}$. Compared to the single-channel the transmission plateau at that energy range approximately to coefficient 1 . The energetically transmission spectra more appeared at the VB state and this shows that more electrons are localized in this area compared to the CB state. This phenomenon is also supported by the bandstructure in Figure 3(b) and DOS in Figure 4(b) for dual-channel APNR since more localized state appeared at VB state.

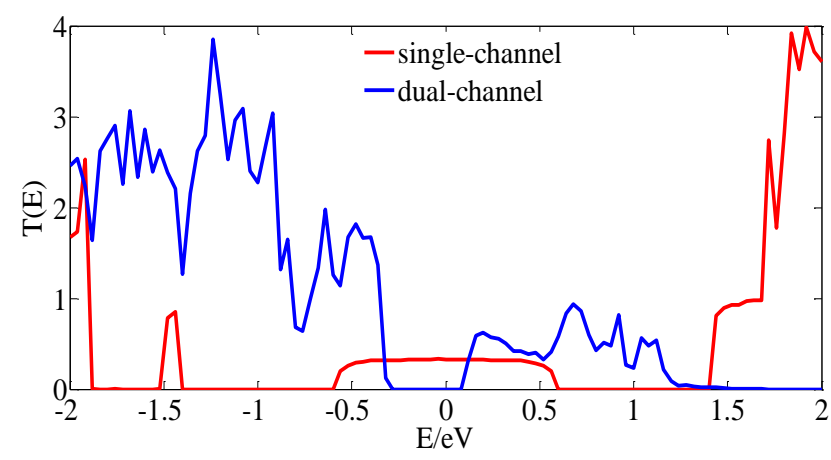

Figure 5. Transmission spectrum for the single-channel and dual-channel APNRs.

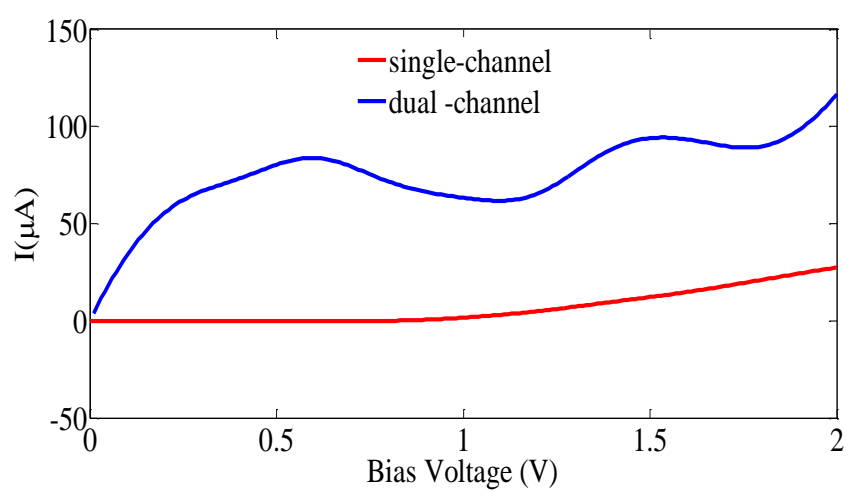

Figure 6. I-V characteristic of single and dual-channel APNRs.

For further evaluation of the transport properties, the current-voltage (I-V) characteristic is simulated. The right electrode was biased from $0.0 \mathrm{~V}$ to $2.0 \mathrm{~V}$ while the left electrode is grounded. The I-V characteristics for APNR for both single and dual channel can be seen in Figure 6 . For single-channel, the current start increasing linearly at the threshold voltage of $0.79 \mathrm{~V}$. The dual-channel behave differently and the current conduction seem to be multiple 
and fluctuating. For example, at $2 \mathrm{~V}$, a great deal of improvement in current can be observed. This in contrast to GNR and CNT where the multiple conducting channel exhibit current to scale linearly with the number of channels $[10,17]$.

\section{CONCLUSION}

A preliminary study on using dual-channels APNR as a channel for transistor has been evaluated particularly on the physical and electronics properties. Through simulation, it is shown that the bandgap decrease when using dual-channels. Furthermore, there are more bands localized in the valence state compared to the conduction state. A suspiring outcome was that the dual-channel APNR generate substantial increment in current at $2 \mathrm{~V}$ compared to single channel. The results obtained in this study suggest the competitiveness of multiple conducting channel of APNR for future electronics device application. This work will stimulate experimental effort to confirm the finding.

\section{ACKNOWLEDGMENT}

Authors would like to acknowledge the financial support under Project Q.J130000/2623.14J08 (GUP Tier 2). Also thanks to the Research Management Center (RMC) of Universiti Teknologi Malaysia (UTM) for providing excellent research environment in which to complete this work.

\section{REFERENCES}

[1] A. Castellanos-Gomez, L. Vicarelli, E. Prada, J. O. Island, K. N. Acharya, S. I. Blanter, D. J. Groenendijk, M. Buscema, G. A. Steele, J. V. Alvarez, H. W. Zandbergen, J. J. Palacios, and H. S. J. Zant, "Isolation and Characterization of FewLayer Black Phosphorus", 2D Materials, vol. 1, no. 2, pp. 025001-025019, 2014.

[2] H. Liu, A. T. Neal, Z. Zhu, D. Omanek and P. D. Ye, "Phosphorene: A New 2D Material with High Carrier Mobility”, ACS Nano, vol. 8, no. 4, pp. 40334041, 2014.

[3] H. Liu, A. T. Neal, Z. Zhu, Z. Luo, X. Xu, D. Tomanek and P.D. Ye, "Phosphorene: An Unexplored 2D Semiconductor with a High Hole Mobility", ACS Nano, vol. 8, no. 4, pp. 4033-4041, 2014.

[4] S. Das, M. Demarteau and A. Roelofs, "Ambipolar Phosphorene Field-Effect Transistor", ACS Nano, vol. 8, no. 11, pp. 11730-11738, 2014.

[5] L. Li, Y. Yu, G. J. Ye, Q. Ge, X. Ou, H. Wu, D. Feng, X. H. Chen and Y. Zhang, "Black Phosphorus FieldEffect Transistors", Nature Nanotechnology, vol. 9, no. 5, pp. 372-377, 2014.
[6] Y. Deng, Z. Luo, N. J. Conrad, H. Liu, Y. Gong, S. Najmaei, P. M. Ajayan, J. Lou, X. Xu, and P. D. Ye, "Black Phosphorus-Monolayer MoS2 Van der Waals Heterojunction p-n Diode", ACS Nano, vol. 8, no. 8, pp. 8292-8299, 2014.

[7] K. -T. Lam, Z. Dong, and J. Guo, "Performance Limits Projection of Black Phosphorus Field-Effect Transistors", IEEE Electron Device Letters, vol. 35, no. 9, pp. 963-965, 2014.

[8] H. Sarvari, C. Liu, A. H. Ghayour, P. Shenavar, Z. Chen and R. Ghayour, "Atomistic quantum transport simulation of multilayer phosphorene nanoribbon field effect transistors", Physica E: Lowdimensional Systems and Nanostructures, vol. 91, pp. 161, 2017.

[9] J. Zhang, H. J. Liu, L. Cheng, J. Wei, J. H. Liang, D. D. Fan, J. Shi, X. F. Tang and Q. J. Zhang, "Phosphorene nanoribbon as a promising candidate for thermoelectric applications", Scientific Reports, vol. 4, pp. 4-11, 2017.

[10] A. D. Franklin, A. Lin, H. P. S. P. Wong, Z. Chen, S. Member, and A. A. Lbg, "Current scaling in aligned carbon nanotube array transistors with local bottom gating," IEEE Electron Device Letters, vol. 31, no. 7, pp. 644-646, 2010.

[11] A. Hellemans, "Ring around the nanowire [news]," Spectrum, IEEE, vol. 50, no. 5, pp. 14-16, May 2013.

[12] Atomistix ToolKit Version 14.2. QuantumWise A/S. (Available: www.quantumwise.com)

[13] Tutorial of using the crystal builder from Quantum Wise (Available :https://docs.quantumwise.com/ tutorials/crystal_builder/crystal builder.html)

[14] Z. Hou and M. Yee. 2007. Electronic and Transport Properties of Graphene Nanoribbons. 7th IEEE Conference on Nanotechnology, (IEEE-NANO) Hong Kong, China. 2-5 August 2007, pp. 554-557, 2007.

[15] A. S. Carmel, A. Pon, R. Ramesh and A. Bhattacharryya, "Calculation of electronic and transport properties phosphorene nanoribbons using DFT and semi empirical models" 2017 International Conference on Microelectronic Devices, Circuits and Systems, ICMDCS 2017, pp. 1-4, 2017.

[16] Q. Wu, L. Shen, M. Yang, Y. Cai, Z. Huang and Y. P. Feng, "Electronic and transport properties of phosphorene nanoribbons",bPhysical Review BCondensed Matter and Materials Physics, vol. 92, no. 3, pp. 1-9, 2015.

[17] Zaharah Johari, A. S. Azman and Razali Ismail, "Feasibility Study of Multiple Channel Armchair Graphene Nanoribbon Field Effect Transistor" 40th International Conference on Micro and Nano Engineering (MNE 2014), Lausanne, Switzerland, 22-26 September 2014. 